\title{
DISCUSSION AFTER PAPER BY VILA
}

Faulkner to Hesser: Can you tell us whether there is any indication of emission features in $C D-42^{\circ} 14462$ ?

Hesser: Yes; $\mathrm{H} \alpha$ is in emission and $\mathrm{H} \beta$ and $\mathrm{H} \gamma$ have weak variable absorption.

Ostriker to Vila: Is it certain that accreting white dwarfs cannot burn $\mathrm{H}$ stably? Is it not possible if $\mathrm{H}$ is accreted sufficiently rapidly? Has this been tried?

Vila: No calculations have been made but I understood that it would not work.

Ostriker: A thermal runaway will occur if burning is in a degenerate region but possibly not if the region is non-degenerate. Such a non-degenerate zone may be produced if the accretion rate is high enough.

Vila: This should be calculated.

Paczyński to Vila: What is the cooling time of a $1 M_{\odot}$ white dwarf until it disappears entirely?

Vila: It is shorter than that for $0.6 M_{\odot}$.

Ostriker: Cooling times are a maximum somewhere in the middle of the white dwarf mass range. At $0.7 M_{\odot}$ the time is about $7 \times 10^{9} \mathrm{yr}$. Above $0.7 M_{\odot}$ and up to $1.4 M_{\odot}$, Debye effects dominate and the time reduces to $10^{9} \mathrm{yr}$. At $0.3 M_{\odot}$ convection dominates and the time is again reduced.

Weidemann to Vila: There is no observational evidence for the existence of either crystallization or liquefaction sequences. John Graham's observations show that the DA white dwarfs form a very well defined and continuous cooling sequence. For the cooler DA's the surface gravity is constan', $\log g=8.3$. For the hotter DA's, the surface gravity might be somewhat lower $(\log g=8)$ as derived by differential comparison for 40 Eri B and from hydrogen line broadening.

Ruben to Hesser: (1) What is the amplitude of the $30 \mathrm{sec}$ variations? (2) Has the star been observed for polarization?

Hesser: The amplitude is very low. No polarization studies have been made. 